Current psychology letters

Contribution of retinal and non-retinal sources of visual information in egocentric object localisation for action

Ludivine Martel, Madeleine Grealy and Yann Coello

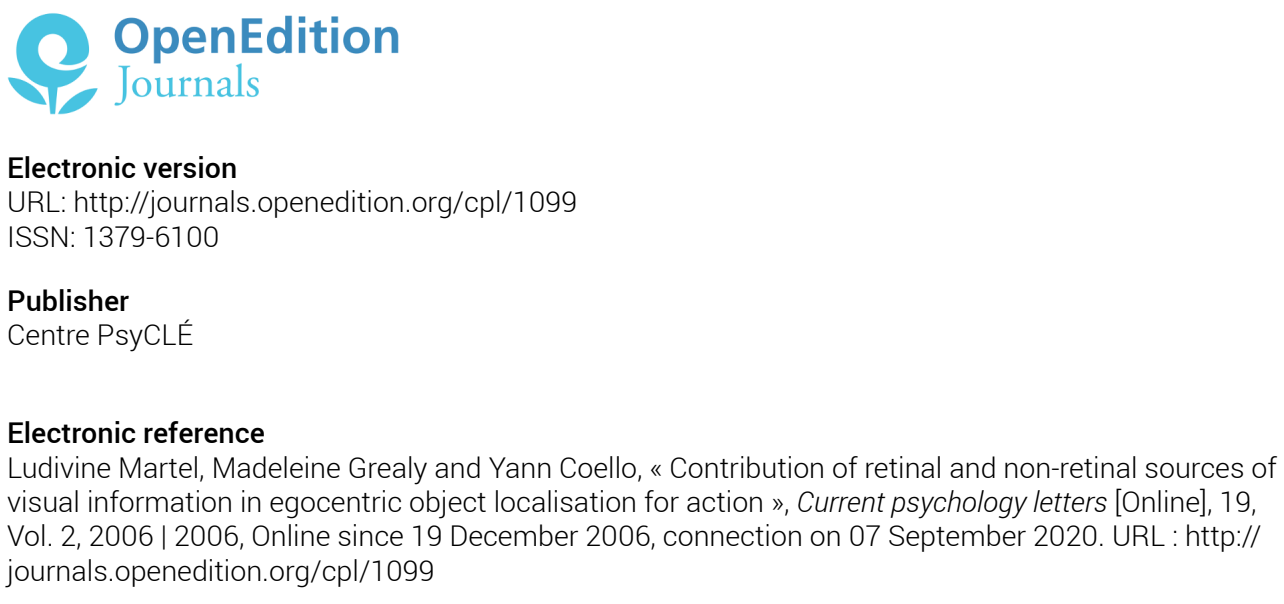

This text was automatically generated on 7 September 2020

(C) All rights reserved 


\title{
Contribution of retinal and non- retinal sources of visual information in egocentric object localisation for action
}

\author{
Ludivine Martel, Madeleine Grealy and Yann Coello
}

Received March 2, 2006

Revised July 30, 2006

Accepted October 3, 2006

On line December 19, 2006

Introduction

Since the pioneering work carried out by Woodworth (1899), a recurrent issue in visuomotor control studies concerns the way visual inputs are integrated to define the location of a visual target in near-body space. In relation to distance coding a large number of experimental works have acknowledged the contribution of two types of visual signals, namely retinal and extra-retinal signals (Blouin et al. 1996, Bock 1986, Servos 2000). Extra-retinal signals refer to information about the position of the eyes obtained from non-retinal sources, including the oculomotor command to displace the fovea towards a visual target (copy of motor efference) and proprioceptive cues transmitted from anatomical structures in the eye muscles. In contrast, retinal signals are independent of eye position and refer to physical aspects of the image that stem from the optical projection of the external world. Though numerous studies have highlighted the participation of extra-retinal cues in eye position coding (e.g. Bridgeman et al. 1991, Treisilian et al. 1999), recent investigations on visuo-manual control have shown that providing a textured workspace, which enhances the retinal information available, brings about a decrease of the perceptual underestimation of distance reported otherwise (e.g. Magne \& Coello 2002, Grealy et al. 2003). For example, substantial underestimation of target location (in general about $10 \%$ of the distance) has been observed when retinal inputs are attenuated due to a reduction in the size of 
the visual scene (Coello \& Grealy 1997), or when the target is presented in a neutral or empty workspace (Foley \& Held 1972). Such distance underestimation suggests that extra-retinal signals do not carry accurate distance information and that there is a constriction of visual space in darkness (Treisilian et al. 1999, Coello \& Iwanow 2006). Thus, visual elements or surfaces structuring the gap between two distant stimuli (namely the hand and the target) may provide some metric information that enables the accurate quantification of the magnitude of the inter-stimuli gap. It is generally acknowledged that retinal information in terms of scene based pictorial cues are ordinal and relative as they are independent of gaze location (Gardner \& Mon-Williams 2001). However, this is not necessary true when the hand is visible in action space as the viewed hand may provide an absolute reference to specify distance from gradient of relative (Brenner et al. 2001) or absolute retinal disparity (Brenner \& Smeets 2006).

Convincing though this argument is, it is not the only possible explanation that should be considered. Other authors have reported that the improvement of target coding due to enhanced retinal signals is not affected by spatial transformation of the retinal inputs. Blouin et al. (2002), for instance, assessed the extent to which retinal inputs affect the accuracy with which participants can indicate their gaze direction after voluntary saccadic eye movements using their unseen index finger. More specifically they tested whether a shift in the retinal image of the environment during the saccade influenced pointing accuracy. These undetected displacements of the visual scene created mismatches between the shift of the retinal image of the environment and the extent of gaze deviation, but the observation that pointing accuracy was not affected led them to suggest that the motor system relies heavily on the use of non-retinal signals. However, the finding that accuracy levels did drop when the task was performed in darkness implied that retinal signals can influence the processing of afferent signals involved in the construction of space (Blouin et al. 1995, 2002). They also suggested that visual stimulation does not necessarily have to convey spatial information relating to the location of a target to be contributive, and concluded that retinal inputs may function in a way that enables extra-retinal signals to be more accurately processed by the central nervous system. In contrast to this though in a subsequent experiment where participants were asked to estimate gaze direction without pointing Blouin et al. (2004) found that estimates of gaze direction were biased by retinal signals to a much greater degree than non-retinal signals. Thus, it appears that retinal information may play different roles for perception and action, with the perception of gaze direction being based on retinal information and the guidance of action being more aligned with extra-retinal signals. Given these surprising results for the involvement of retinal and extra-retinal cues, the aim of the present study was to investigate this further and to consider the importance of the contributory nature of the context in which the target is set. The methodology consisted of decoupling the retinal and extra-retinal signals by the means of vertically deviating optical prisms (10 dioptres, i.e. 5.4 degrees). Such methodology has been used in the past to probe the sources of information contributing to distance coding. Gardner and Mon-Williams (2001) for instance used similar perturbation paradigm and reported that vertical prisms affected the perceived distance of target in a pointing task. In particular, bottom-up prismatic deviation caused participants to overshoot target position, suggesting that vertical gaze angle can be used as a distance cue. However, when analysing distance judgments under the prismatic displacement of the whole visual scene, the retinal hand-to-target gap was not considered in this study. This was mainly 
due to the fact that the responding arm was out of view when participants estimated the target location, and this has been shown to provide crucial information when judging the location of a visual target following prisms exposure (Redding \& Wallace 1996). Furthermore, only one (structured) visual context and only one (binocular) viewing condition was investigated.

The present study aims to evaluate the respective role of retinal and extra-retinal signals in distance coding in a more complete experimental design and when vision of the whole action space is made available. Visual targets were presented (monocularly or binocularly) in darkness or on a horizontal textured background whilst wearing neutral (0 dioptre) or prismatic (10 dioptres) spectacles. Displacing the horizontal visual surface by 10 dioptres has the consequence of modifying extra-retinal signals (as the gaze is oriented 5.4 degrees further away for similar target location) whilst leaving retinal signals virtually unchanged. Thus, reaching for a distant target should be significantly affected by the prismatic deviation only if extra-retinal signals prevail in providing more veridical distance information on which to base the action.

MethodsParticipants

Ten self-declared volunteers and right-handed students from the University of Lille3 participated in the experiment (mean age 22 years). All participants had normal or corrected vision (contact lens) and were naïve as to the purpose of the experiment. They all gave their informed consent prior to their inclusion in the experiment, which was approved by University Charles de Gaulle ethical committee.

Apparatus and procedure

The experimental apparatus consisted of a rectangular box $(60 \mathrm{~cm}$ high, $100 \mathrm{~cm}$ wide and $70 \mathrm{~cm}$ deep) with the inside divided horizontally by a half-silvered mirror. A computer 20 " monitor was placed upside-down on the top surface of the apparatus so that the image generated by the computer was reflected in the mirror, and due to the optical geometry, the image on the computer screen appeared to project onto the bottom surface. Three targets (green dots, diameter $10 \mathrm{~mm}$ ) were randomly presented along the sagittal axis at $178 \mathrm{~mm}, 228 \mathrm{~mm}$ and $278 \mathrm{~mm}$ from the starting location of the hand. Each target was presented either in darkness or with a textured background made with grey dots of $5 \mathrm{~mm}$ randomly positioned over the whole workspace $(30 \mathrm{~cm} \times 39 \mathrm{~cm})$. Internal surfaces of the box were smooth and painted matt black and no visual information from the external environment was available during the experimental session (see Figure 1). The vision of the target and the hand at the starting position was available from a focussed dim light beam, but there was no visual feedback about arm displacement. The participants were instructed as to which target to point to as quickly and accurately as possible. No instruction was given about the form of the movement trajectory.

All participants performed 10 pointing movements towards each of the targets in two vision conditions (monocular or binocular), two prism conditions $\left(0^{\circ}\right.$ or $\left.5.4^{\circ}\right)$ and two background conditions (darkness or textured background). A $5.4^{\circ}$ deviation of the visual scene was obtained by bottom-up 10 dioptres prismatic spectacles, and neutral spectacles $\left(0^{\circ}\right)$ were used for control performances. The order of presentation of the experimental conditions was counterbalanced across the participants, and each person completed a total of 240 trials, with blocks of 10 trials for each type of conditions ( 2 visual contexts $\mathrm{x} 2$ vision conditions $\mathrm{x} 2$ optical conditions $\mathrm{x} 3$ distances $\mathrm{x} 10$ trials).

Data recording and processing 
Horizontal pointing movements were performed on a digitizer tablet (Wacom UD-185, spatial resolution: $0.1 \mathrm{~mm}$ ) which registered the $(\mathrm{x} ; \mathrm{y})$ coordinates of an electromagnetic stylus held in the participant's right hand (sample rate: $100 \mathrm{~Hz}$ ). Endpoint positions of individual movements were used to compute constant and variable terminal errors. Constant error was decomposed into radial error (the distance between movement vector length and the target vector length, with a minus sign to indicate an undershoot) and angular error (the angle between the starting-position-to-endmovement-position vector, with a minus sign to indicate a deviation to the right of the target). The distance of the target to which movements were referenced was based on the spectacles used so that with no deviation of the visual scene the target distances were $178 \mathrm{~mm}, 228 \mathrm{~mm}$ and $278 \mathrm{~mm}$, whereas with the 10 dioptres deviation of the visual scene the distance between the hand and the target optically increased by $8.73 \mathrm{~mm}$, $13.85 \mathrm{~mm}$ and $20.29 \mathrm{~mm}$ (for the targets located at $178 \mathrm{~mm}, 228 \mathrm{~mm}$ and $278 \mathrm{~mm}$ respectively, see Figure 1). Thus, in the presence of prismatic deviation, gaze direction moved on average $5.6 \mathrm{~cm}$ away for similar target locations, whereas the retinal hand-totarget gap increased by only $1.4 \mathrm{~cm}$ on average.

For the purposes of analysis uncorrected data and corrected data (allowing for slight variations in hand-to-target gap induced by the prismatic spectacles) were successively analysed. Kinematic (peak velocity) and temporal (reaction time, movement time, percentage taken by the acceleration period) parameters were examined from the hand path. A three-way analysis of variance (ANOVA: background (2) $x$ vision condition (2) $x$ prisms condition (2)) was performed with repeated measures on all the factors using the corrected data. In the case the sphericity assumption was violated (i.e. Epsilon smaller than 1), Huyn-Feldt adjustments of the p-values were reported.

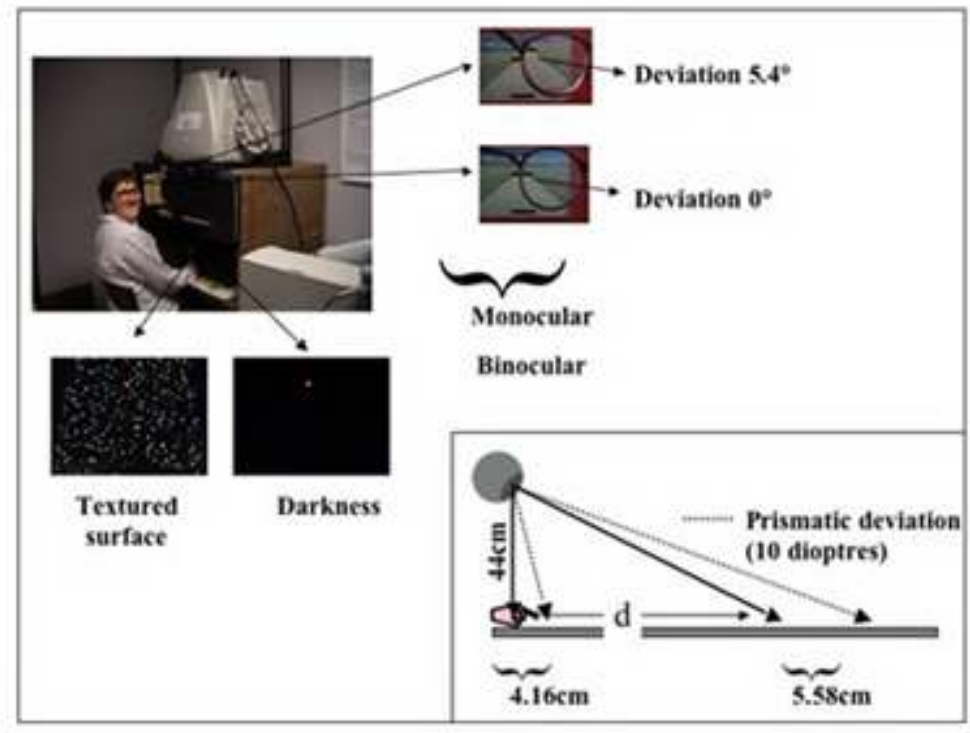

Figure 1. Apparatus and experimental factors (Vision condition : binocular-monocular; Prisms condition : 0 - 10 dioptres; Background condition : darkness, textured surface). In the lower part is shown the mean increase of target distance due to the optical displacement of the whole visual scene.

ResultsSpatial performance

With regards to spatial accuracy, the radial error $(-9.70 \mathrm{~mm}$ on average, SD: $41.42 \mathrm{~mm})$ was weakly influenced by the visual condition $(F(1,9)=4.15, p=0.07$, with binocular 
condition:-7.04mm (SD: $39.03 \mathrm{~mm}$ ) and monocular condition: $-12.38 \mathrm{~mm}$ (SD: $43.52 \mathrm{~mm}$ )). However, an interaction between the background and prisms conditions was observed $(F(1,9)=6.48, p=0.03)$. This was due to the fact that radial error was lower with prisms spectacles than with neutral spectacles but only in darkness as shown by the simple effects $(F(1,9)=14.64, p<0.01$ and $F(1,9)=0.22, p=0.65$, respectively in the darkness and textured background condition, with $0^{\circ}:-25.67 \mathrm{~mm}(S D: 34.88 \mathrm{~mm})$ and $1.48 \mathrm{~mm}$ (SD: $40.84 \mathrm{~mm}$ ), $5.4^{\circ}:-14.21 \mathrm{~mm}$ (SD: $37.62 \mathrm{~mm}$ ) and $-0.44 \mathrm{~mm}$ (SD: $37.31 \mathrm{~mm}$ ) respectively). Further analysis showed that these effects were not affected by target location $(F(2,9)=3.68, p=0.82)$ though the general hypometry reduced with distance (close target mean error: $-17.65 \mathrm{~mm}$ (SD: $40.04 \mathrm{~mm}$ ), middle target: $-10.82 \mathrm{~mm}$ (SD: $47.94 \mathrm{~mm}$ ), far target: $-1.72 \mathrm{~mm}$ (SD: $53.71 \mathrm{~mm})$ ). Furthermore, since no feedback was provided during the experiment, the radial error did not change from the five first (mean=-9.08mm) to the five last trials (mean $=-11.27 \mathrm{~mm}, F(1,9)=1.21, p=0.30$ ). The angular error was, on average, $2.60 \mathrm{deg}$ (SD: $3.27 \mathrm{deg}$ ) to the left and was not influenced by the background $(F(1,9)=4.04, p=0.08)$, the prisms $(F(1,9)=0.59, p=0.46)$ or the visual condition $(F(1,9)=1.35$, $p=0.28$ ). Mean trajectories for the half-way target shown in Figure 2 demonstrate the findings of this spatial analysis. It is worth mentioning that in darkness, the raw effect of the prismatic spectacles was an increase of movement amplitude of $22.41 \mathrm{~mm}$ (SD: $33.11 \mathrm{~mm}$ ) and $26.19 \mathrm{~mm}$ (SD: $33.27 \mathrm{~mm}$ ) in the binocular and monocular visual conditions respectively.

Temporal performance

Overall mean movement duration was $725 \mathrm{~ms}$ (SD: 231ms) and there was no significantly difference between the monocular and binocular vision conditions $(F(1,9)=1.65, p=0.2)$. It was, however, greater with the prisms (744ms, SD: 242ms) than the neutral spectacles (706ms, SD: $220 \mathrm{~ms}, F(1,9)=13.08, p<0.01$ ), which could be due to the small increase in movement amplitude that occurred when wearing the prisms as shown by the raw data. There is a tendency for overall duration to be significantly influenced by the background condition $(F(1,9)=4.49, p=0.06$, with $759 \mathrm{~ms}$ (SD: $257 \mathrm{~ms}$ ) and $690 \mathrm{~ms}$ (SD: $198 \mathrm{~ms}$ ) for the textured background and darkness conditions respectively). No interaction between the variables was observed. Furthermore, the percentage of acceleration phase according to the total movement duration was 53.32\% (SD: 4.89\%). This percentage was not significantly influenced by the visual condition $(F(1,9)=0.72$, $p=0.42)$, the background $(F(1,9)=0.02, p=0.90)$ or the prisms $(F(1,9)=0.65, p=0.44)$. The peak velocity was smaller with the neutral spectacles $(619 \mathrm{~mm} / \mathrm{s}, S D: 303 \mathrm{~mm} / \mathrm{s})$ compared to the prisms $(646 \mathrm{~mm} / \mathrm{s}, S D: 268 \mathrm{~mm} / \mathrm{s})$, but this difference did not reach significance $(F(1,9)=2.94, p=0.12)$. Additionally, peak velocity was not influenced by the vision $(F(1,9)=0.11, p=0.75)$ or the background conditions $(F(1,9)=0.13, p=0.73)$. Finally, reaction time was $399 \mathrm{~ms}$ ( $S D: 249 \mathrm{~ms}$ ) on average and was influenced by neither the background $(F(1,9)=0.08, p=0.78)$, the visual condition $(F(1,9)=0.41, p=0.54)$ or the prisms $(F(1,9)=0.67, p=0.43)$. 


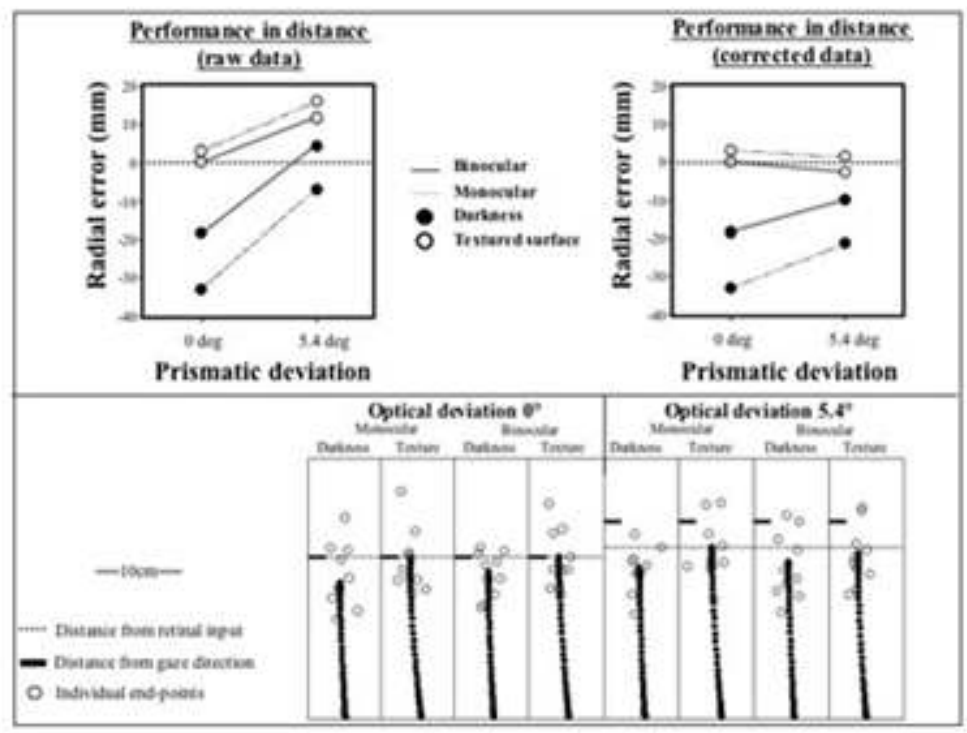

Figure 2. Upper part shows the distance performance for the raw and corrected data (i.e. after subtracting for the slight increase in target distance with the10 dioptres prisms). In the lower part is shown the mean trajectory and individual end-points for the 10 participants when pointing to the target located at $228 \mathrm{~mm}$ in the different prismatic conditions.

Discussion and conclusion

The aim of the present study was to assess whether the benefit in distance coding seen in a visuo-motor task when a textured workspace is provided was due to an elaboration of the retinal signals giving more veridical distance information or an enhancement in the use of the extra-retinal signals thought to be used to guide the action. The rational was that optical deviation produced by the way of prisms should affect performance only if reaching to the target location was guided by extra-retinal signals since the whole visual scene was displaced, leaving the retinal structure of the visual input virtually unchanged. Furthermore, the influence of the retinal signals, if any, should be more obvious in presence of a textured workspace. An analysis of pointing accuracy showed that once the slight variation of the visual hand-to-target gap due to the optical effect of the prismatic deviation (6\% of target distance on average) was taken into account, no significant variations in distance performance were found when the workspace was textured. The estimated distance of the target was indeed accurate and similar in presence or absence of the prismatic deviation (mean radial error was $-0.44 \mathrm{~mm}$ and $1.48 \mathrm{~mm}$ respectively), despite the $5.4^{\circ}$ of change of gaze direction that the optical deviation induced (corresponding on average to $5.6 \mathrm{~cm}$ in the workspace). This lack of a prismatic effect is understandable only if one assumes that the spatial information used to guide the action originated from the retinal signals. Indeed, with the gaze being directed $5.4^{\circ}$ further away with the prismatic spectacles than the actual location of the target, an involvement of the extra-retinal signals in target coding would have induced a significant increase in movement amplitude (5.6 $\mathrm{cm}$ in theory). This suggests that in presence of a structured environment the increase in accuracy of reaching distance estimation is due to a prevalence of spatial information on the retina. These results are in line with previous observations, where structuring the workspace instantaneously improves distance perception (Foley et al. 1972, Magne \& Coello 2002). It furthermore indicates that structuring the visual scene enables access to more 
veridical distance information rather than it playing a gating function that enables extra-retinal signals to be further and more accurately processed (Blouin et al.1995, 2002). It is possible that since the hand was visible on the textured background this provided some egocentric visual cue that enabled a more accurate interpretation of familiar scene based pictorial cues or gradient of absolute retinal disparity. It is indeed acknowledged that if a familiar object is visible, somewhere in the scene, we could use its image size to determine the scaling factor for relative distances (Brenner et al. 2001). The positive effect of structuring the retinal signals was indeed more prominent in the present study than in previous one that limited the visual scene to the target area (e.g. Gardner \& Mon-Williams 2001). It would be interesting to further consider this assumption in future experimental work. Interestingly, the present data suggest that texture cues may improve distance coding independently on the presence or not of stereoscopic vision, as distance performance was as accurate in the binocular and the monocular vision condition when a textured background was available. Thus, though horizontal (Howard \& Rogers 1995) and vertical (Brenner et al. 2001) retinal disparities participate in depth perception, the lack of binocular information did not seem to prevent accurate distance coding, as this was already described with other psychophysical paradigm such as slant surface perception for instance (Bergman \& Gibson 1959). This is in agreement with the idea that binocular vision mainly improves people's everyday movements by giving them a better sense of the distances of relevant objects, rather than by relative retinal disparities being used to directly guide the movement (Brenner \& Smeets 2006). This also suggests that a calibration of extraretinal information used to estimate vertical gaze angle from the gradient of retinal disparity (Gardner \& Mon-Williams 2000) is not a sufficient interpretation to account for all our data. Though it is difficult to interpret findings in particular in presence of a limited set of conditions, they rather suggest that distance coding may accurately stem from the combination of the position of various cue images on the retina, including the hand, the target and the texture (absolute retinal disparities), that could be scaled using familiar size or egocentric location of the visual hand.

It is also worth mentioning that providing a textured workspace affected mainly the perceptual system as no variations of the intrinsic properties of the motor responses were found when wearing the prisms. Though movement duration slightly increased when pointing with the prismatic spectacles (probably due to the optical extent of target distance), the proportion of the acceleration (53\% on average) and deceleration ( $47 \%$ on average) phases were not influenced by the experimental manipulations. Thus, the coding of the distance of the target seemed to be predominately based on an offline estimation of the hand-to-target gap given by retinal information. The lack of difference between the monocular and binocular visual conditions in terms of the amplitude of the movements when the visual scene was structured is in agreement with this interpretation.

In the same vein, the poorer estimates of distance seen in darkness, that is in the absence of any visual cues relating to the workspace but the hand, indicated that crucial information was missing to properly encode the location of the target. This again suggests that extra-retinal signals do not carry accurate distance information, at least when the visual space is not calibrated by the retinal signals, as has already been suggested in the past (e.g. Brenner \& Van Damme 1998, Magne \& Coello 2002). The distance of the target was broadly underestimated $(-2.57 \mathrm{~cm}$ in absence of optical deviation which corresponded to $11 \%$ of the target distance), and even more so in the 
monocular $(-3.3 \mathrm{~cm})$ than the binocular $(-1.83 \mathrm{~cm})$ condition. The tendency to underestimate distance suggests a constriction of the visual space in darkness and consequently the presented targets appeared nearer than their actual location. This kind of constriction is supported by the findings of a previous study where the limit to which visual targets appeared to be reachable receded in darkness with the same magnitude than characterises the underestimation as when pointing to them (Coello \& Iwanow 2006). The opposite effect, but of same magnitude, indicated that in both situations the targets were perceived to be closer than their actual location. Furthermore, a strong effect of the prismatic spectacles on the distance performance was observed in the present study $(2.6 \mathrm{~cm}$ considering the raw data). This indicates that in darkness participants seemed to refer with more confidence to their extra-retinal signals in order to encode target location. This interpretation is in agreement with the increasing confidence in vergence as a cue to distance in sparse environments that has been reported in previous studies when position coding was tested at eye level (e.g. Treisilian et al., 1999). This also suggests that the angular hand-to-target separation from the retinal image combined with the eventual felt position of the hand, is not enough to code distance properly.

Though the present data argue in favour of a prevalence of retinal information in distance coding in a natural viewing context, there might be one way to reconcile the controversial interpretations that were suggested in the past by dissociating the various spatial dimensions. It might be that distance represents a particular spatial dimension, which necessitates a greater contribution of retinal information to compensate for the lack of accuracy of vergence and/or vertical gaze angle signals (Magne \& Coello 2002). Indeed, when the visual targets are presented along the horizontal axis, which focuses the task on directional coding, visuo-motor performance seems to rely more on extra-retinal sources of information to locate targets (Blouin et al. 1995, 2002). In contrast, presenting the targets along the sagittal axis seems a more complex situation as errors in locating gaze direction have more dramatic effect on spatial performance in the near-far than the right-left dimension (Brenner \& Smeets 2000). Thus, additional sources of information are required and obviously these are embedded within the geometrical structure of the surfaces delimiting the hand-totarget gap.

\section{BIBLIOGRAPHY}

Bergman R, Gibson JJ. (1959). The negative after-effect of the perception of a surface slanted in the third dimension. American Journal of Psychology, 72, 364-374.

Blouin J, Amade N, Vercher JL, Teasdale N, \& Gauthier GM. (2002). Visual signals contribute to the coding of gaze direction. Experimental Brain Research, 144, 281-92.

Blouin J, Bresciani JP, \& Vercher JL. (2004). Shifts in the retinal image of a visual scene during saccades contribute to the perception of reached gaze direction in humans. Neural Letters, 357, 29-32. 
Blouin J., Gauthier G.M., \& Vercher J.L. (1995). Internal representation of gaze direction with and without retinal inputs in man. Neural Letters, 183, 187-189.

Blouin J., Gauthier G.M., Vercher J.L., \& Cole J. (1996). The relative contribution of retinal and extraretinal signals in determining the accuracy of reaching movements in normal subjects and a deafferented patient. Experimental Brain Research, 109, 148-153.

Bock, O. (1986). Contribution of retinal versus extraretinal signals towards visual localization in goal-directed movements. Experimental Brain Research, 64, 476-482.

Brenner, E. \& Smeets, J.B.J. (2000) . Comparing extra-retinal information about distance and direction. Vision Research, 40, 1649-1651.

Brenner E., Smeets J.B.J., \& Landy M. (2001). How vertical disparities assist judgement of distance. Vision Research, 41, 3455-3465.

Brenner E. \& Smeets J.B.J. (2006) . Two eyes in action. Experimental Brain Research, 170 302-311.

Brenner E. \& Van Damme W.J.M. (1998). Judging distance from ocular convergence. Vision Research, 39, 493-498.

Bridgeman B. \& Stark L. (1991). Ocular proprioception and efference copy in registering visual direction. Vision Research, 31, 1903-1913.

Coello Y. \& Grealy M.A. (1997). Effect of size and frame of visual field on the accuracy of an aiming movement. Perception, 26, 287-300.

Coello, Y. \& Iwanow, O. (2006). Effect of structuring the workspace on cognitive and sensorimotor distance estimation: no dissociation between perception and action. Percept Psycho, (in press).

Foley, J.M. \& Held, R. 12 (1972).Visually directed pointing as a function of target distance, direction, and available cues. Percept Psycho, 263-268.

Gardner, P.L. \& Mon-Williams, M. (2001). Vertical gaze angle: absolute height-in-scene information for the programming of prehension. Experimental Brain Research, 136 379-385.

Grealy, M.A., Coello, Y., \& Heffernen, D. (2003). Constructing visual space from apparent motion distorts the perception of object location. Experimental Brain Research, 150, 356-362.

Howard IP. \& Rogers BJ. (eds.)(1995). Binocular vision and stereopsis. Oxford; Oxford University Press.

Magne, P., \& Coello, Y. (2002). Retinal and extra-retinal contribution to position coding. Behavioural Brain Research, 136, 277-287.

Redding, G.M. \& Wallace, B. (1996). Adaptive spatial alignment and strategic perceptual-motor control. Journal of Experimental Psychology: Hum Percept Perform, 22, 379-394.

Servos P. (2000). Distance estimation in the visual and visuomotor system. Experimental Brain Research, 130, 35-47.

Tresilian J.R., Mon-Williams M., \& Kelly B.M. (1999). Increasing confidence in vergence as a cue to distance. Proc $R$ Soc Lond B, 266, 39-44.

Woodworth R.S. (1899). The accuracy of voluntary movement. Psychological Review, 3 1-114. 


\section{ABSTRACTS}

In visuo-manual tasks, enhancing visual information by presenting a target-object on a textured background has been proved to improve distance coding. However, controversial interpretations have suggested that enriched retinal inputs either provides access to more veridical spatial information or enables extra-retinal signals (e.g. vergence) to be more accurately processed. To contrast these interpretations, the present study evaluated the spatial accuracy of upper limb movement towards visual targets presented monocularly or binocularly either in darkness or on a textured background. Action was performed whilst wearing neutral or prismatic spectacles deviating by $5.4^{\circ}$ gaze direction but leaving spatial information on the retina virtually unchanged. We found that distance underestimation observed in darkness reduced in presence of a textured background whatever the vision condition. Surprisingly, the performance was not affected by prismatic displacement when a textured background was provided. These results indicate that distance processing for action relies mainly on retinal inputs in presence of a structured workspace, even when there is a mismatch between retinal and extra-retinal signals.

Dans les tâches visuo-manuelles, enrichir les informations visuelles en présentant un objet cible sur une surface texturée permet d'optimiser le traitement de la distance égocentrée de cet objet. Toutefois, des interprétations différentes de ce phénomène ont été proposées, suggérant que l'accroissement des stimulations rétiniennes permettrait soit un traitement plus véridique de la distance égocentrée à partir des indices rétiniens picturaux, soit un traitement plus précis des signaux extra-rétiniens comme la vergence. Pour confronter ces deux interprétations, nous avons évalué la précision spatiale d'un déplacement segmentaire vers des cibles visuelles perçues en vision binoculaire ou monoculaire dans l'obscurité ou bien en présence d'une surface texturée. Les cibles étaient perçues à travers des lunettes neutres $\left(0^{\circ}\right)$ ou prismatiques déviant la direction $d u$ regard $\left(5,4^{\circ}\right)$ sans modifier significativement la structure des informations rétiniennes. Les résultats mettent en évidence une sous-estimation de la distance dans l'obscurité qui diminue fortement en présence d'une surface texturée indépendamment des conditions de vision. De manière surprenante, lorsqu'une surface texturée est présente les performances ne sont pas affectées par la déviation prismatique. Ces résultats indiquent que le traitement de la distance pour l'action s'appuie principalement sur la structure des informations rétiniennes, même en présence d'une discordance manifeste entre signal rétinien et signal extra-rétinien.

\section{AUTHORS}

\section{LUDIVINE MARTEL}

URECA, Department of Psychology, University of Lille III, France 